\title{
Ranking Method for Mediators in Social Network
}

\author{
Ryosuke Saga and Hiroshi Tsuji \\ Graduate School of Engineering \\ Osaka Prefecture University \\ 1-1 Gakuen-Cho, Naka-ku, Sakai, Japan 5998531 \\ saga@mis.cs.osakafu-u.ac.jp, tsuji@cs.osakafu-u.ac.jp
}

\begin{abstract}
This paper proposes a method for ranking mediators where a mediator is defined as node having an important role in a social network. To precisely rank the mediators in order of their importance, a method is used based on changes in the average shortest path length. However, the computational complexity for this method is $\mathrm{O}\left(\mathrm{N}^{5}\right)$, so an unreasonable amount of time it is required to determine complexity for a massive network. Our ranking method, whose complexity is no more than $\mathrm{O}\left(\mathrm{N}^{2}\right)$, is based on the relationships among adjacency nodes. Although the method does not provide a precise but an approximate rank, we found that there is a strong correlation between the ranks generated using the strict and the developed methods. Results on a variety of generated networks confirmed the feasibility of our method for a massive network.
\end{abstract}

Keywords: Social Network, Mediator, Rank Mining, Community Computing, Graph Theory.

\section{Introduction}

Let us imagine the following organization: members openly share their knowledge and exchange ideas not only within groups but also with groups outside the organization. Additionally, members within the organization work cooperatively with external experts and other skilled persons to assist and support each other. This type of organization is called an open innovation organization [1]. Intel, IBM, etc. have succeeded in actively making open innovation organizations.

Within an organization, a social network can exist and consists of nodes and links in which each node indicates a person and each link shows the relationship of the dyad. If an organization is expressed as a social network, the central people in the network are called leaders, and many people work around the leaders. However, to express plural organizations which work collaboratively in open innovation, each company is required to have mediators who communicate outside the borders of organizations. Such people are not always leaders but are sometimes considered just as important as leaders. In order to manage and drive such mediators, it is necessary to find and rank them, and the method to rank mediators uses changes in the average shortest path length. However, the method has a problem that it takes a lot of time to compute the method because of large complexity. 
In this paper, we evaluated a new method for ranking a mediator's importance. Our fast approximation method is based on the relationship among adjacency nodes of potential mediators. The approximation method focuses on local nodes, even though a strict method is required to handle all the nodes within a network.

The reminder of this paper is structured as follows: Section 2 is a literature review of the importance of mediators and their influence to a network. Section 3 outlines two ranking methods: a strict method, which uses the average shortest path, and our approximation method. We compared the strict method with our method. Section 4 checks feasibility of our method. Section 5 discusses related and future works. Finally, we conclude this paper in Section 6.

\section{Role of Mediator}

If a mediator leaves an enterprise described as open innovation organization, the enterprise will lose some critical communication paths and some nodes will fail to collaborate. Moreover, the associated enterprise connected by the mediator will also not be able to work effectively either and will need to invest additional costs to compensate their weak points. The cost may be wasted, and the related enterprises may incur additional costs when working out the costs. Therefore, mediators can be thought as influencing an entire network.

According to research about patents and inventors Fleming et al. [2], reported that some inventors play a role of gatekeeper and make innovation more progressive in a network. Such inventors can be regarded as a mediator in a network. In the management science area, Malone et al. [3] proposed a team form called the X-team. Mediators have the same importance as key decisions makers in the X-team who cooperate with other related projects. Leydesdorff [4] indicated that new developments may occur at the borders of disciplines, that is, interdisciplinary relationships are formed. Girvan et al.[5] shows that any group connected to each other through a small number of mediators in a collaborative network Research has shown that large internet service providers seldom connect directly each other [6], which highlights the importance of mediators.

Any mediator plays an important role in their organization, and therefore, it is important to develop a method to manage and drive mediators. Ranking mediators based on their existence value for management is an effective method for manage. Consequently, we evaluated, as described in the following section two ranking methods: strict method based on the average shortest path length and our approximation method based on the links created by adjacency nodes.

\section{Ranking Methods}

In the following sections, we assumed that a link has neither direction nor weight.

\subsection{Strict Solution}

Mediators affect not only adjacency nodes but also the entire network. The degree of influence is high when a node is removed from a network and affects the network path length. This is because mediators exist in the shortest path between specific nodes. 
Accordingly, we focused on the change in the average shortest path length. Note that the average shortest path length is the average of the distance, $d_{i j}$, between two arbitrary nodes called node $i$ and node $j$ in a network where $N$ nodes exist. Distance $d_{i j}$ is the number of the required links to connect node $i$ and node $j$. We can get the average length, $L_{i}$, of node $i$ by using formula (1):

$$
L_{i}=\frac{1}{N-1} \sum_{i} d_{i j}
$$

Calculating $L_{i}$ for all nodes, we can calculate the average shortest path length in the network by using formula (2):

$$
L=\frac{1}{N} \sum_{i} L_{i}
$$

By using the average shortest path length in a network, we can assign mediators' ranks as follows:

1. Remove a node individually,

2. Calculate the average shortest path for each, and

3. Measure the change in length and rank them.

However, there is a problem about computational complexity. Under the premise that Dijkstra's algorithm [7] is used to compute the distance between nodes. Assuming that Dijkstra's algorithm, which is a representative method, is used to compute the distance, formula (1) needs $\mathrm{O}\left(\mathrm{N}^{3}\right)$ because the complexity of Dijkstra's algorithm is $\mathrm{O}\left(\mathrm{N}^{2}\right)$. For computing formula (2), the computational complexity becomes $\mathrm{O}\left(\mathrm{N}^{4}\right)$. Additionally, this process should be repeated for all nodes. As a result, the final complexity is $\mathrm{O}\left(\mathrm{N}^{5}\right)$ in practice. Therefore, this method is impractical for a massive network.

\subsection{Proposed Method: Approximation Method}

To highlight the difference between the methods, we will describe a local structure and the relationships formed. Fig. 1(a) and Fig. 1(b) indicate the importance of the node. These figures represent situations when node $v$ has the same number of adjacency nodes in a dense network with a focus on node $v$ and another less dense network. Even if node $v$ is removed from the network, the effect on adjacency nodes is small because there are many alternative links. Therefore, node $v$ can be regarded as an unimportant mediator. Contrary to this, assuming that the relationship among adjacency nodes is weak, as shown in Fig. 1(b), several adjacency nodes would not able to communicate when node $v$ is removed from the network. In this case, the role of node $v$ as a mediator is considered more important than in the preceding case.

From the above features, we hypothesize the following:

Hypothesis 1. Nodes whose adjacency node has a small number of direct links to others have higher importance than mediators than other nodes.

Hypothesis 2. The importance of a mediator is indicated by the importance of its adjacency nodes.

For the first hypothesis, let us consider Fig. 1(a). We can regard node $v$ as an unimportant mediator because the numbers of links from adjacency nodes to other nodes 
do not change even when node $v$ is removed. On the other hand, the numbers of links from adjacency nodes to other nodes shown in Fig. 1(b) are less than those shown in Fig.1 (a). This means that node $v$ shown in Fig. 1.(b) can be regarded as a more important mediator than node $v$ shown in Fig.1.(a).

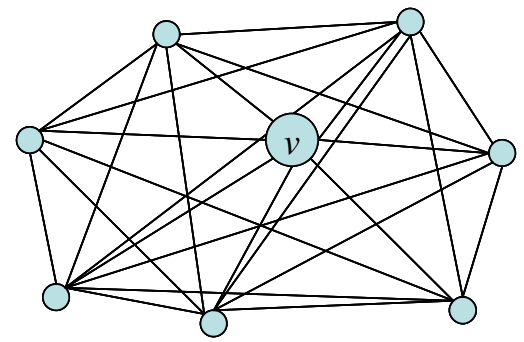

(a) Dense Network with a focus on Node v

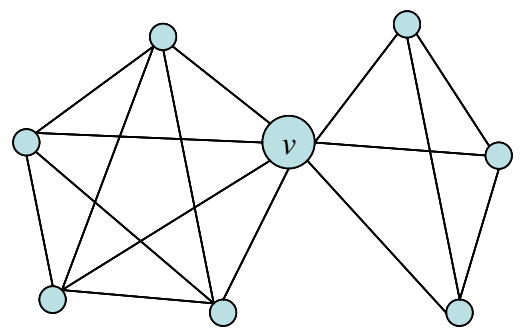

(b) Nondense Network with a focus on Node $\mathrm{v}$

Fig. 1. Relation among node $v$ and its adjacency nodes

For the second hypothesis, the effect of the neighbors' influence is made highlighted by several methods. For example, the concept of PageRank assumes that a web page linked by important pages is also important [7]. Thus, we consider that the degree of importance a mediator has reflects the influence of adjacency nodes.

Consequently, we can compute a node's rank for a mediator based on the two hypotheses. We assume that node $v$ has adjacency nodes $v_{i}$ (where $\left.\mathrm{i}=1, \ldots ., n\right)$ in which the number of its links is $E_{i}$ and define $r_{i}$ as the short number of links for node $v$ as compared to a complete graph. When node $v$ is removed from the network, $b_{i}$, which links the decline rate of node $v_{i}$, is given as follows:

$$
b_{i}=\frac{r_{i}}{n-1}=\frac{n-1-E_{i}}{n-1} .
$$

As a result, $B_{v}$ which is a mediator score of node $v$, is shown as follows:

$$
B_{v}=\sum_{i=1}^{n} b_{i}=n-\sum_{i}^{n} \frac{E_{i}}{n-1}
$$

Arranging the values shown in formula (4) in descending order, we can obtain the node rank for each mediator.

Next, we will consider the computational complexity of our method. Our algorithm needs the most computational complexity when a network creates a complete graph because formula (4) needs $\mathrm{O}(\mathrm{N})$ per each node. Therefore, the total complexity is $\mathrm{O}\left(\mathrm{N}^{2}\right)$, which is by far smaller than previous strict methods $\mathrm{O}\left(\mathrm{N}^{5}\right)$.

\subsection{Simple Example}

The difference between the two methods can be shown using a simple example, like that network shown in Fig. 2 We will initially show the example using the strict method. Table 1 lists the distances necessary to create the average shortest path length about Node0. Note that when the distance is 10 , a node cannot reach another node. 
The average shortest path of Node 0 is 5.41, as listed in Table 1. Finally, the computed values and ranks are listed in Table 2.

When our method is used, the value of Node0 is computed as follows:

$$
6-\frac{0+0+0+2+2+2}{6-1}=4.8 \text {. }
$$

For iteration of our method in the same way as the strict method, the values of the nodes are listed in Table 3. Thus, our method indicates that a node is zero if adjacency nodes of the node under review form a complete graph within themselves.

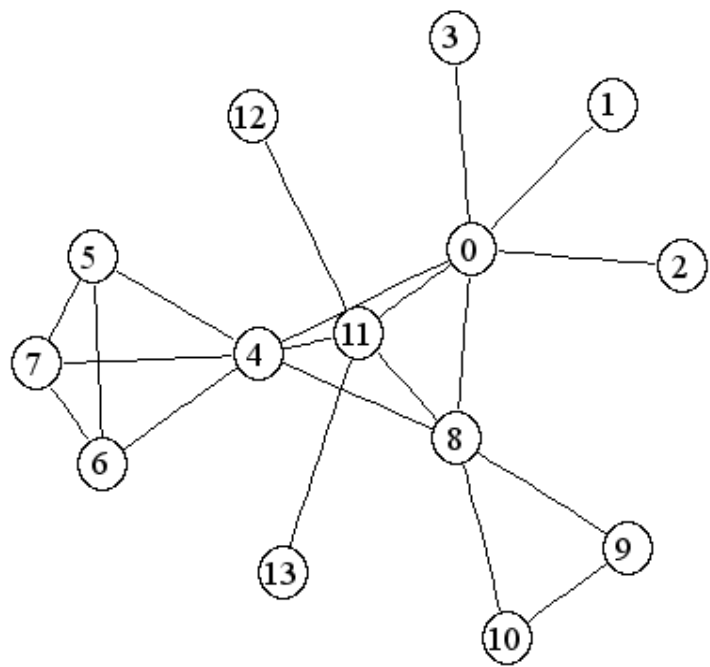

Fig. 2. Example of simple network having 14 nodes

Table 1. The number of hops when node 0 is removed. (When a node is not reachable to another, the value is 10 .).

\begin{tabular}{|c|c|c|c|c|c|c|c|c|c|c|c|c|c|}
\hline $\begin{array}{c}\text { Node } \\
\text { (From } \backslash \text { To) }\end{array}$ & 1 & 2 & 3 & 4 & 5 & 6 & 7 & 8 & 9 & 10 & 11 & 12 & 13 \\
\hline 1 & & 10 & 10 & 10 & 10 & 10 & 10 & 10 & 10 & 10 & 10 & 10 & 10 \\
\hline 2 & 10 & & 10 & 10 & 10 & 10 & 10 & 10 & 10 & 10 & 10 & 10 & 10 \\
\hline 3 & 10 & 10 & & 10 & 10 & 10 & 10 & 10 & 10 & 10 & 10 & 10 & 10 \\
\hline 4 & 10 & 10 & 10 & & 1 & 1 & 1 & 1 & 2 & 2 & 1 & 2 & 2 \\
\hline 5 & 10 & 10 & 10 & 1 & & 1 & 1 & 2 & 3 & 3 & 2 & 3 & 3 \\
\hline 6 & 10 & 10 & 10 & 1 & 1 & & 1 & 2 & 3 & 3 & 2 & 3 & 3 \\
\hline 7 & 10 & 10 & 10 & 1 & 1 & 1 & & 2 & 3 & 3 & 2 & 3 & 3 \\
\hline 8 & 10 & 10 & 10 & 1 & 2 & 2 & 2 & 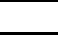 & 1 & 1 & 1 & 2 & 2 \\
\hline 9 & 10 & 10 & 10 & 2 & 3 & 3 & 3 & 1 & & 1 & 2 & 3 & 3 \\
\hline 10 & 10 & 10 & 10 & 2 & 3 & 3 & 3 & 1 & 1 & 7 & 2 & 3 & 3 \\
\hline 11 & 10 & 10 & 10 & 1 & 2 & 2 & 2 & 1 & 2 & 2 & & 1 & 1 \\
\hline 12 & 10 & 10 & 10 & 2 & 3 & 3 & 3 & 2 & 3 & 3 & 1 & & 2 \\
\hline 13 & 10 & 10 & 10 & 2 & 3 & 3 & 3 & 2 & 3 & 3 & 1 & 2 & \\
\hline
\end{tabular}


Table 2. Values and ranks of strict method

\begin{tabular}{|l|c|c|r|r|r|r|r|r|r|r|r|r|r|c|}
\hline Node & \multicolumn{1}{c|}{1} & 1 & 2 & 3 & 4 & 5 & 6 & 7 & 8 & 9 & 10 & 11 & 12 & 13 \\
\hline Value & 5.41 & 2.14 & 2.14 & 2.14 & 5.1 & 2.17 & 2.17 & 2.17 & 4.33 & 2.14 & 2.14 & 4.44 & 2.13 & 2.13 \\
\hline Rank & 1 & 8 & 8 & 8 & 2 & 5 & 5 & 5 & 4 & 8 & 8 & 3 & 13 & 13 \\
\hline
\end{tabular}

Table 3. Values and ranks of proposed method

\begin{tabular}{|l|r|r|r|r|r|r|r|r|r|r|r|r|r|r|}
\hline Node & \multicolumn{1}{c|}{1} & 1 & 2 & 3 & 4 & 5 & 6 & 7 & 8 & 9 & 10 & 11 & 12 & 13 \\
\hline Value & 4.8 & 0 & 0 & 0 & 3.6 & 0 & 0 & 0 & 3 & 0 & 0 & 3.5 & 0 & 0 \\
\hline Rank & 1 & 5 & 5 & 5 & 2 & 5 & 5 & 5 & 4 & 5 & 5 & 3 & 5 & 5 \\
\hline
\end{tabular}

\section{Experiment}

\subsection{Network Model}

We compared the strict method and our method experimentally. Initially, we checked the rank correlation between the two methods using generated random networks, and then measured the computation time.

In Random networks in the first experiment were generated as follows:

Step1. Make $N$ nodes where $N$ is $20,30, \ldots$ or 100.

Step2. Divide them into $M$ groups and connect the links between nodes in the same group. Reduce the size of $M$ from 5 to $M / 7$, and each group has mi nodes based on a normal distribution where the average equals $N / M$ and a variance of 2 is used.

Step3. Connect groups like a ring network and add random $L$ links between selected nodes. Note that $L=N * t, \mathrm{t}$ ranges from 0.1 to 1 , and the distance is set to infinity if there is no path between nodes.

The sample network is shown in Fig. 3.

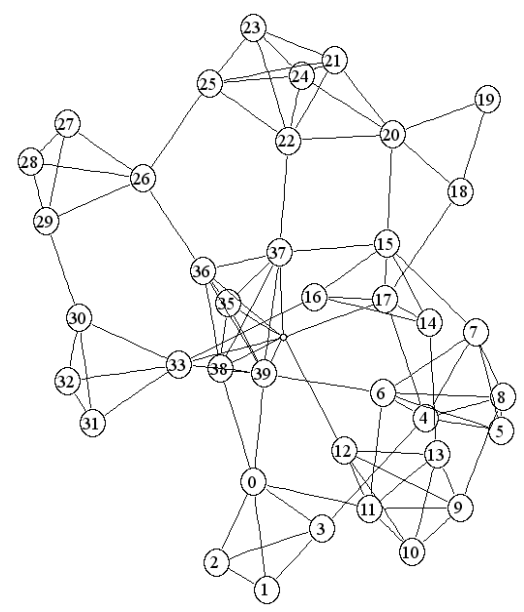

Fig. 3. Generated sample network having 40 nodes 
The second experiment involving computation time was when we produced networks consisting of nodes $(N)$ and density $(D)$ (that is, the networks have links of $\left.D^{*} N^{*}(N-1) / 2\right)$. The number of nodes ranged from 20 to 300 , and the density ranged from 0.1 to 1 (that is, a complete graph).

\subsection{Result}

Table 4 lists and Fig. 4 shows the correlation of rank between the strict method and our approximation method. We found a strong correlation that was greater than 0.8 for most cases. We also found that the correlation does not depend on the $t$ value.

Table 4. Correlation between node and rate of random link

\begin{tabular}{|c|c|c|c|c|c|c|c|c|c|c|}
\hline tlNode & 20 & 30 & 40 & 50 & 60 & 70 & 80 & 90 & 100 & Average \\
\hline 0 & 0.87 & 0.86 & 0.85 & 0.84 & 0.86 & 0.88 & 0.82 & 0.85 & 0.87 & 0.86 \\
\hline 0.1 & 0.83 & 0.86 & 0.84 & 0.85 & 0.83 & 0.80 & 0.83 & 0.81 & 0.85 & 0.83 \\
\hline 0.2 & 0.18 & 0.83 & 0.79 & 0.70 & 0.81 & 0.78 & 0.84 & 0.77 & 0.81 & 0.75 \\
\hline 0.3 & 0.81 & 0.75 & 0.84 & 0.81 & 0.88 & 0.81 & 0.85 & 0.89 & 0.73 & 0.82 \\
\hline 0.4 & 0.87 & 0.81 & 0.83 & 0.77 & 0.90 & 0.72 & 0.91 & 0.90 & 0.79 & 0.84 \\
\hline 0.5 & 0.84 & 0.85 & 0.74 & 0.81 & 0.68 & 0.81 & 0.90 & 0.81 & 0.82 & 0.81 \\
\hline 0.6 & 0.76 & 0.79 & 0.84 & 0.92 & 0.90 & 0.84 & 0.77 & 0.93 & 0.78 & 0.84 \\
\hline 0.7 & 0.87 & 0.81 & 0.88 & 0.76 & 0.76 & 0.88 & 0.81 & 0.78 & 0.81 & 0.82 \\
\hline 0.8 & 0.73 & 0.77 & 0.90 & 0.83 & 0.78 & 0.96 & 0.83 & 0.90 & 0.73 & 0.82 \\
\hline 0.9 & 0.66 & 0.71 & 0.86 & 0.82 & 0.95 & 0.92 & 0.94 & 0.93 & 0.93 & 0.86 \\
\hline 1 & 0.57 & 0.82 & 0.96 & 0.76 & 0.82 & 0.70 & 0.87 & 0.77 & 0.75 & 0.78 \\
\hline Average & 0.73 & 0.81 & 0.84 & 0.80 & 0.83 & 0.82 & 0.85 & 0.84 & 0.82 & 0.82 \\
\hline
\end{tabular}

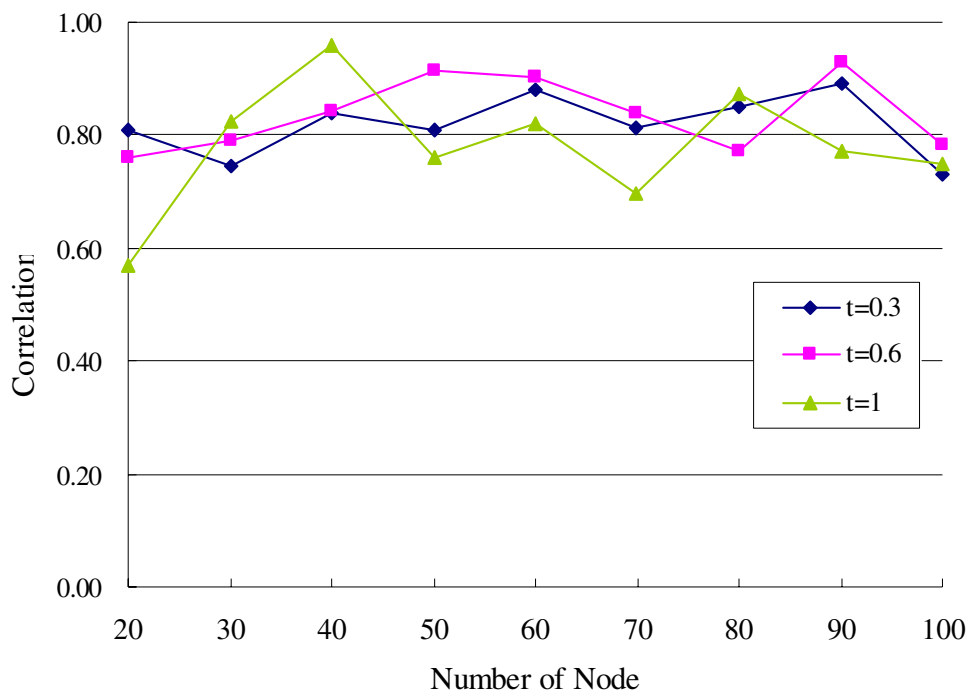

Fig. 4. Correlation when the rate of additional link $t$ is $0.3,0.6$ and 1 
The results of the computational time are shown in Fig. 5. Note that only one case is shown for the strict method because the results are similar for each other. The results in this figure show that our method reduced the computation time when compared to the strict method. We understand that our method depends on density. For example, the computation time in networks of density 1 is more than 10 times of that in the networks of density 0.1 .

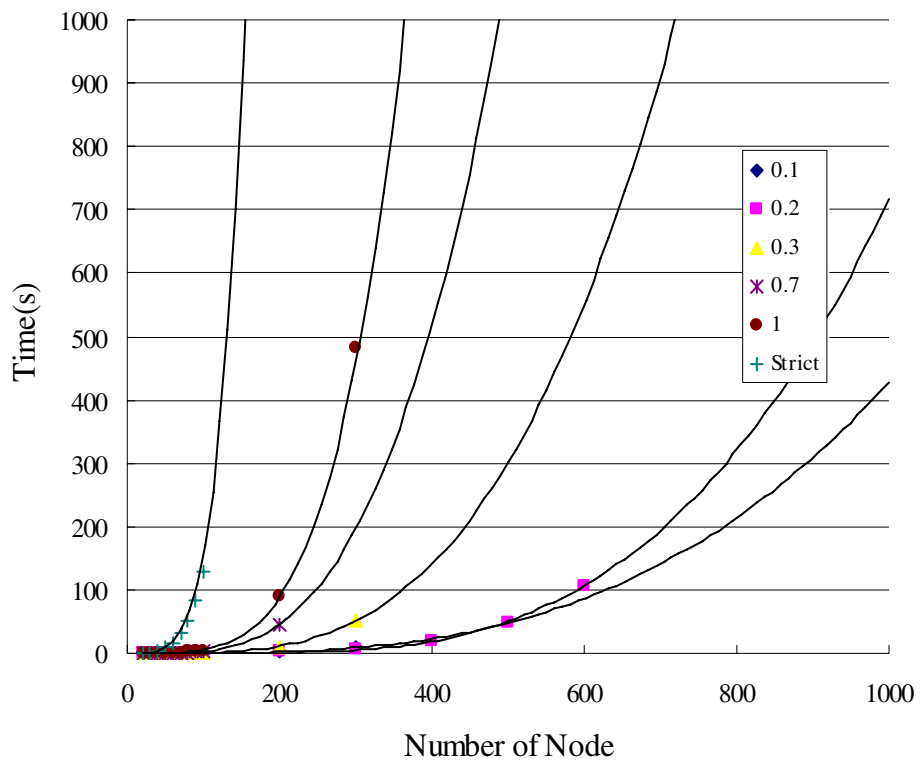

Fig. 5. Time consumption among strict method and approximation methods

\section{Discussion and Future Work}

Various centrality methods: closeness centrality, degree centrality, and betweenness centrality, have already been proposed to rank nodes [9].

Closeness centrality is an index that considers a node important when the distances among nodes is short. Degree centrality is an index that considers a node important when the nodes have many adjacency nodes. However, these indices measure centrality and do not measure the rank of the mediator.

On the other handWhereas, betweenness centrality is an index that determines the importance of each node based on the actual number of shortest paths between vertices it has. This index is also useful when ranking mediators. However, all the network data need when measuring them globally. Thus, computational complexity used to measure betweenness centrality is well-known as $\mathrm{O}\left(\mathrm{N}^{3}\right)$. Therefore, the betweenness centrality is not appropriate measure for massive networks in terms of time complexity. Additionally, one can argue that processing is possible when there are many short paths in a network, even though the concept of betweenness centrality is relatively easy to understand. 
Our method ranks nodes based on the local relationship among adjacency nodes. Thus, the method is able to compute a node's ranks even when not all network information is known and is also able to minimize complexity $\mathrm{O}\left(\mathrm{N}^{2}\right)$. However, we consider that arguments about precision and correlation and betweenness centrality have not been discussed in the current research. This is a future work in our research.

\section{Conclusion}

We developed an approximation method to rank mediators. Initially, we introduced a strict method and highlighted the problem of computational complexity. To solve this complexity, we developed a method based on the relationships among adjacency nodes. By comparing our method and the strict method, we found that a strong correlation exists between the results produced by each test and that our method can reduce computation time drastically.

\section{References}

1. Chesbrough, H.W.: Open Innovation, Harvard Business School Press (2003)

2. Fleming, L., Adam, J.: A Network of Invention, Harvard Business Review, vol. 82(4) (2004)

3. Malone, T.W.: The Future of Work: How the New Order of Business Will Shape Your Organization, Your Management Style, and Your Life. Harvard Business School Press, Boston, MA (2004)

4. Loydesdorff, L.: "Betweenness Centrality" as an Indictor of the "Interdisciplinarity" of Scientific Journals, Journal of the American Society for Information Science and Technology (forthcoming) (2007)

5. Girvan, M., Newman, M.E.J.: Community structure in social and biological networks, Proc. Natl. Proc. Natl. Acad. Sci. USA 99, 8271-8276 (2002)

6. Pastor-Satorras, R., Vazquez, A., Vespignani, A.: Dynamical and correlation properties of the internet, Phys. Rev. Lett. vol. 87(25) (2001)

7. Dijkstra, E.W.: A note on two problems in connection with graphs. Numerische Mathematik 1, 269-271 (1959)

8. Google: http://www.google.com

9. Freeman, L.C.: Centrality in social networks: Conceptual clarification. Social Networks 1, 215-239 (1979) 